\title{
Persistence and reversibility of arsenic-induced gut microbiome and metabolome shifts in rats after 30 - days recovery duration
}

\section{Xiaoyan Du}

Institute of Urban Environment Chinese Academy of Sciences https://orcid.org/0000-0003-1108-955X

Jie Zhang ( $\sim$ jie.zhang@xmu.edu.cn )

Institute of Urban Environment Chinese Academy of Sciences https://orcid.org/0000-0002-5902-3317

\section{Karl-Werner Schramm}

Helmholtz Center Munich-German Research Center for Environmental Health, Molecular EXposomics

\section{Qingyu Huang}

Institute of Urban Environment, Chinese Academy of Sciences

\section{Meiping Tian}

Institute of Urban Environment, Chinese Academy of Sciences

\section{Xi Zhang}

Institute of Urban Environment, Chinese Academy of Sciencesme

\section{Bingru Nan}

Institute of Urban Environment, Chinese Academy of Sciences

\section{Heqing Shen}

Institute of Urban Environment, Chinese Academy of Sciences

\section{Research}

Keywords: Arsenic, Gut microbiome, Metabolome, Reversibility.

Posted Date: May 29th, 2020

DOI: https://doi.org/10.21203/rs.3.rs-29645/v1

License: (1) (1) This work is licensed under a Creative Commons Attribution 4.0 International License. Read Full License 


\section{Abstract}

Background: The metabolites of gut microbiome are important host-health regulating factors and can be easily interrupted by the host exposure to environmental pollution via ingestion route. Arsenic contaminated drinking water is one of the most serious environmental problems worldwide. Therefore, the arsenic-induced alterations of gut microbiome and the metabolome, especially the persistence and reversibility parts of the alterations after the long-term arsenic exposure will be interesting to know.

Results: We investigated the relationship between gut microbiota and its related metabolites in male rats both after the 30-days arsenic treatment and 30-days recovery duration. The composition and diversity of gut microbiota were affected significantly by the treatment, but they presented partial improvement in recovery duration. Moreover, arsenic exposure induced the significant changes of 73 metabolites, which involved in the metabolism of glycerophospholipid, linoleic acid, as well as the biosynthesis of phenylalanine, tyrosine and tryptophan. Although it had a persistent effect, the restoration of glycerophospholipid metabolism was observed in the 30-days recovery. Integration analysis further correlated the arsenic impacting microbes with some important differential metabolites, but only Lactobacillus associated with the decreases of phosphatidylethanolamine(34:1) , 16alphahydroxydehydroepiandrosterone 3-sulfate, seryltryptophan and alanyltyrosine in the recovery. Lactobacillus strains have potential to work as protective agents against arsenic toxicity by restoring perturbed glycerophospholipid metabolism.

Conclusions: Arsenic significantly modified gut microbiome and metabolome, but arsenic-induced disruptions of gut microbiome and metabolome are reversible to some extent after a 30-days recovery. The deeper understanding of correlation of altered gut microbiome and metabolome could be a novel strategy to combat arsenic-induced disease.

\section{Background}

Many areas of the world are at high risk of drinking arsenic-contaminated water. ${ }^{1}$ Lots of studies indicate that chronic exposure to arsenic associated with a variety of complications, such as integumentary, nervous, respiratory, cardiovascular, immune, endocrine and reproductive system. ${ }^{2,3}$ The etiologies of dysfunctional metabolism, altered lipid deposition and chronic inflammation are similar to the disease progression of chronic change in gut microbiome. ${ }^{4-6}$

Lots of clinical and animal studies demonstrate the critical role of the gut microbiota in both health maintenance and disease pathogenesis. ${ }^{6-8}$ The gut microbiota is involved in the regulation of multiple host metabolic pathways, thereby resulting in interplay host-microbiota metabolic, signalling, and immune-inflammatory axes. ${ }^{9}$ Therefore, altered gut microbiome can cause damage and disease in other organs, and the microbiome has come to the forefront as a reflection of many diseases. ${ }^{10}$ Various evidences indicate that the gut microbiome could be perturbed by arsenic. ${ }^{11-13}$ In addition, because gut microbiome is deeply involved in host metabolism, raising the concern of arsenic exposure could have 
the interplay with microbiome to impact host health. For example, arsenic-induced significant changes of the abundance of Firmicutes and Bacteroidetes are tightly related to the lipid metabolites, indolecontaining metabolites, isoflavone metabolites, and bile acid metabolites. ${ }^{12}$ These evidences demonstrate that arsenic exposure induced energy metabolism disorder, immune response and low inflammation through altering gut microbiome composition, and consequently led to metabolic disease. $14-15$

The gut microbiome is a very complicated dynamic system. Restoration of the gut microbiome may happen partially or fully after removing the treated-condition. Many studies find microbiome change are partially reversible from a high-fat, low-fat or high-sugar diet to the original diet. ${ }^{16-18}$ Similarly, in six weeks after 10-days cefoperazone treatment, the community structure of the gut metabolome associate with the state of $C$. difficile susceptibility differed from that encountered in non-antibiotic-treated mice. ${ }^{19}$ However, there is very little evidence for the recovery of gut microbiome after terminating heavy metal exposure. In fact, lots of people lived in the arsenic-contaminated areas in a short time and no longer exposed to arsenic after their departure. Therefore, the arsenic-induced alteration is possible to be persistent even after the withdrawal of the treatment, or the alteration is also possible to be restored after a recovery period. Up to now, previous studies focus on the arsenic-induced alteration of the gut microbiota, but it is not clear whether its exposure could impact on the later development. Furthermore, it is not still known whether these effects are reversible after the end of arsenic exposure.

The integrated analysis of microbiome and metabolome can help to identify the previously unrecognized mechanisms of arsenic-induced lesion on the relation of gut microbiota to metabolite difference, in further may uncover the reversibility of arsenic-induced microbiome effects in gut, and the later can be clinical important. To answer the effects lasting after the individual getting rid of the exposure, the study was designed on the weaned male rats. We applied an integrated approach by combining 16S rRNA sequencing technique and untargeted metabolomics to determine the effects of arsenic on the gut microbiome and its metabolite profiles via water drinking exposure, and the recovery of these effects after terminating arsenic exposure were investigated.

\section{Results}

\section{Alterations in the gut microbiota diversity}

The rats were divided into ten groups representing 30-days control group, four As-treated groups, 60-days control group and four As-recovery groups (Fig. 1). In our microbiome analysis, the sampling depth was adequate (Additional file 1: Result S1). The Chao1 and ACE index increased remarkably in both As-treated and As-recovery groups. There were also significant changes for the Shannon and Simpson indexes in As-treated groups (Fig. 2a, 2b; Additional file 2: Table S1), while most of them were not significantly changed in As-recovery groups with the exception of the Shannon indexes in the $0.05 \mathrm{mg} / \mathrm{L}$ and $6.25 \mathrm{mg} / \mathrm{L}$ As-recovery groups. Significant difference was observed in $\beta$-diversity, both the weighted and unweighted principal coordinates analysis (PCOA) plots revealed the separation between As-treated 
groups and As-recovery ones based on the first two PCoA (Fig. 2c, 2d). However, As-recovery groups clustered closer to their control group than As-treated groups. These results suggest that the diversity of gut microbiota was strongly affected by arsenic, whereas the impact can be mostly recovered.

Figure 1. Experiment diagram. Eighty weaned male Wister rats (PND 23) were randomly divided into ten groups ( $\mathrm{n}=8$ per group). The rats in 30-days control group (PND 53) and 60-days control group (PND 83) received deionized water alone. Four group rats (As-treated groups) were treated with 0.05, 0.25, 1.25 and $6.25 \mathrm{mg} / \mathrm{L}$ arsenic trioxide (ATO) via water drinking route for 30 days, respectively. The other four group rats (As-recovery groups) were the animals that firstly treated with the upper mentioned doses of ATO for 30-days and then with the deionized water for the additional 30-days.

Figure 2. Gut microbiome diversity and structure analysis. Species diversity differences were estimated by the Chao1 and ACE (a), Shannon and Simpson (b). PCoA plot base of the relative abundance of the operational taxonomic units (OTUs) (97\% similarity level) showing bacterial structural cluster. (c) Weighted unifrac PCoA plots; (d) Unweighted unifrac PCoA plots. ${ }^{\star} p<0.05 ;{ }^{* \star} p<0.01$.

\section{Alterations in the gut microbiota composition}

Seventeen phyla were identified, and listed in Table S2, Additional file 2. Arsenic altered both structure and composition of the gut microbiota. Firmicutes, Proteobacteria and Bacteroidetes were predominant in the gut microbiota of arsenic-treated rats at the phylum level (Fig. 3). Other phyla groups including Actinobacteria, Verrucomicrobia and Gemmatimonadetes were detected at low levels. In As-recovery groups, the main bacteria are still Firmicutes, Proteobacteria and Bacteroidetes. Welch's t-test was performed to test the significance of the three phyla's alteration (Fig. 4). The taxonomic assignments showed Firmicutes decreased significantly, whereas Proteobacteria and Bacteroidetes increased significantly (Additional file 2: Table S3). The relative proportions of the three phyla in As-recovery groups were similar to those in related controls.

In addition, arsenic exposure increased the abundance of some phyla, such as Clostridia, Clostridiales, and Bacteroidetes (Fig. 5a). After 30-days recovery, some phyla primarily showed the higher enrichment than control, such as Lactobacillus, Lactobacillaceae, Lactobacillales and Bacilli (LDA score $(\log 10)>4.8$ and $\mathrm{P}<0.05$ ) (Fig. 5b). 42 discriminatory OTUs were identified from the microbiota of As-treated groups (Additional file 1: Figure S2a), in which 34 are specific OTUs on the results of LEfSe and listed in Table S4a, Additional file 1. On their abundance variations, the taxonomies were sufficient to differentiate the gut microbiota in these rats when exposed to arsenic. 79 discriminatory OTUs were identified from the Asrecovery groups (Additional file 1: Figure S2b), while 22 specific OTUs were identified on the results of LEfSe (Additional file 2: Table S4b).

Figure 3. The gut microbiome composition profiles at the phylum level. Firmicutes, Proteobacteria and Bacteroidetes were predominant in the gut microbiota of rats at the phylum level.

Figure 4. Welch's t-test results for evaluating significance of the relative abundance for the three predominant microbiotas at the phylum level. Firmicutes decreased significantly, whereas Proteobacteria 
and Bacteroidetes increased significantly after arsenic exposure. The relative proportions of the three phyla in As-recovery groups were similar to those in 60-days control group.

Figure 5. Linear discriminant analysis (LDA) integrated with effect size (LEfSe). The abundance discriminated and administration-specific OTUs in the As-treated groups (a) and the As-recovery groups (b).

\section{Alterations in gut metabolome}

An untargeted metabolome approach was used to explore the gut metabolite alteration that associated with the treatment. Arsenic exposure perturbed the metabolic profiles in gut, which consisted of both microbiota and rat metabolites. Orthogonal partial least squares discriminant analysis (OPLS-DA) showed the clear distinctions between the As-treated groups and 30-days control group, as well as Asrecovery groups and 60-days control group based on the first two principal components (Additional file 1: Figure S3), suggesting arsenic-induced specific metabolomic alterations. A total of 80 differential metabolites were identified (Additional file 2: Table S5). The number of differential metabolites in Asrecovery groups were significantly reduced compared to As-treated groups (Additional file 1: Figure S4), which implied that most of the metabolomics alteration in rat gut are restorable after the 30-days recovery.

The alteration of gut metabolome in As-treated groups was characterized by the significant changes of lipids, amino acids, peptides, nucleotides (Fig. 6). These metabolites are mostly involved in glycerophospholipid metabolism, linoleic acid metabolism, tryptophan metabolism, as well as phenylalanine, tyrosine and tryptophan biosynthesis (Fig. 7), suggesting that these pathways are sensitive to arsenic exposure in the microenvironment of rat gut.

Only a few of differential metabolites in As-recovery groups were observed, and most of them were not significantly changed in As-treated groups. The differential metabolites in As-recovery groups were significantly enriched in glycerophospholipid and purine metabolism, glycosylphosphatidylinositol (GPI)anchor biosynthesis, as well as pentose and glucuronate interconversions (Fig. 7). The metabolic networks (Fig. 8) generated on the differential metabolites, and the TOP 10 differential metabolites (Additional file 2: Table S6) that mostly associated with the other ones were selected marked. In addition, the metabolites that related to the significantly affected pathways are shown in Table S7, Additional file 2 .

Figure 6. Heatmap showing the differentially accumulated and significantly changed metabolites in the As-treated groups and As-recovery groups. ${ }^{*} p<0.05 ;{ }^{* \star} p<0.01$.

Figure 7. Radar map of gut microbial metabolic pathway-enrichment analysis of differential metabolites in the As-treated groups and As-recovery groups.

Figure 8. Metabolic correlation networks of metabolic correlation network of the As-treated groups (a) and the As-recovery groups (b). TOP 10 differential metabolites that mostly associated with the other ones 
were tagged.

\section{Association of the gut microbial dysbiosis with metabolism dysregulation}

Spearman correlation analyses were conducted for arsenic-specific gut microbiome and the selected differential metabolites, which included the upper mentioned TOP 10 differential metabolites and the metabolites that related to the significantly affected metabolic pathways. Figure 9a shows that some metabolites such as lysoPC(20:4), PC(36:4), lysoPE(15:0) and DG presented positive correlation with most of the specific gut microbiome in As-treated groups, especially xylanophilum_group (OTU000192), Lachnospiraceae (OTU000285, OTU000391), Ruminococcaceae_NK4A214_group (OTU000276) and Ruminococcaceae (OTU000309, OTU000358). On the contrary, MIA, Indole, PE(38:4), 19,20-DiHDPA, CS, MG(16:1), MG(20:5), 11'-CGT3 and lysoPC(20:3) were negatively correlated with the OTUs mentioned above, as well as Bacteroidales_S24-7_group (OTU000395), Clostridium_sensu_stricto_1 (OTU000335), Lachnospiraceae_NK4A136_group (OTU000547), Ruminiclostridium_5(OTU000517) and coprostanoligenes_group (OTU000209). In As-recovery groups, the strength of the relationship between gut microbiome and metabolites (Fig. 9b) was significantly reduced. Increased lysoPC(15:0) and decreased PE(34:1), 16a-HEA-3S, STP, Ala-Tyr were mainly implicated in the Lactobacillus (OTU000600, OTU000608, OTU000842 and OTU001536).

Figure 9. Spearman's rank correlation between the specific gut microbial species and the differential metabolites for the As-treated groups (a) and the As-recovery groups (b).

\section{Discussion}

Previous studies suggest that the microbiome and metabolome of the gut content could be affected by arsenic. ${ }^{11,12}$ The present study not only demonstrates more comprehensive evidences for the arsenic impact on gut microbiome and metabolome, but also shows the partial reversibility of the effects after the 30 -days recovery duration.

\section{Arsenic induced gut microbiome and its metabolic profile alterations in rats}

The microbiome data clearly shows that arsenic exposure induced the profound changes in rat gut microbiome, namely the changes of composition and diversity of the microbiota. Firstly, the microbial diversity significantly increased and this seems to be a counterintuitive situation, because the higher diversity is usually thought as the healthier in microbiota. However, the risk of malnutrition has been associated with the increased microbiota diversity. ${ }^{20}$ Therefore, it is not sure these changes are supportive for health or not. The disturbances of gut microbiota by arsenic may induce some diseases. Under arsenic stress, taxonomic assignments of Firmicutes decreased, while Proteobacteria and Bacteroidetes significantly increased. The changes of these phyla and increased bacterial diversity have 
been observed in the feces from the children with autism. ${ }^{21}$ The gut microbiota was more diverse in the autism spectrum disorder (ASD) children than in the control group, ${ }^{22,23}$ which also may explain the counterintuitive result of our study. In addition to the gut microbiome changes, the arsenic levels in the ASD children also increases significantly, ${ }^{22}$ indicating the gut microbiome disruption may be one of the potential mechanisms of arsenic-induced autism.

The liver is a major target organ for arsenic toxicity. ${ }^{24}$ Arsenic concentration $\geq 0.3 \mathrm{mg} / \mathrm{L}$ in drinking water significantly increase risk of hepatitis or cirrhosis in people without chronic viral hepatitis. ${ }^{25}$ Liver disorders have been associated with microbiome disruption. ${ }^{26}$ Compared to healthy people, lower Firmicutes and higher Bacteroidetes, Proteobacteria proportions were detected in the faeces of nonalcoholic steatohepatitis patients. ${ }^{27}$ Moreover, increased Proteobacteria abundance is even a remarkable maker of liver cirrhosis. ${ }^{28,29}$ The same change trends of these bacteria species were also observed in the rats exposed to arsenic. In our study, certain arsenic treatment increased the levels of liver injury markers (i.e. serum total protein TP, albumin ALB, total bile acid TBA and lactic dehydrogenase LDH) (Additional file 1: Figure S5), indicating the potential association between arsenic exposure, microbiome disruption and hepatic injury.

The altered gut microbiota is also a potential indicator of cardiovascular disease (CVD) ${ }^{30}$ Bacterial metabolic products can penetrate intestinal barrier into systemic circulation, and induce low-grade chronic inflammation and elevate CVD risk. ${ }^{31}$ Arsenic exposure is one of the common causes of CVD. ${ }^{32,33}$ The elevated biochemical parameters of serum LDH, alpha-hydroxybutyric dehydrogenase (a-HBDH) and creatine kinase MB isoenzyme (CK-MB) in this study suggested the possibility of myocarditis or myocardial infarction (MI) after arsenic exposure (Additional file 1: Figure S5). Previous evidences show that the disordered gut microbial communities determine the susceptibility to $\mathrm{MI}$ in rats and human. ${ }^{34,35}$ In this study, treatment groups presented increased Proteobacteria, which is consistent with the findings from the patients with $\mathrm{Ml}^{36}$ Gut microbes, through trimethylamine N-oxide generation, directly contribute to enhance the risk of platelet hyperreactivity and thrombosis, such as MI. ${ }^{37}$ There are similar results for an elevation of Proteobacteria in myocarditis and atherosclerosis. ${ }^{38,39}$ Moreover, the high level of Proteobacteria was associated with atherosclerotic plaques, ${ }^{40}$ thus it was speculated that the arsenicinduced elevated Proteobacteria may have pro-inflammatory effects and further contribute to plaque progression. These evidences increase the possibility of gut microbiome to predict arsenic-induced CVD risk.

In order to uncover the metabolic information behind arsenic-induced microbiome alteration, we performed untargeted analysis of gut microbial metabolome. Lachnospiraceae is an important gut microbial species and suspected to be a specific indicator of arsenic-induced gut bacteria imbalance. Lachnospiraceae significantly associated with the disruption of lipid metabolism. The specie has positive correlation with phosphatidylcholine metabolites, such as lysoPC (20:4) and lysoPE (15:0), which also could promote CVD. ${ }^{5,41}$ Additionally, as important components of cell membranes, phospholipids play critical roles in maintaining cell membrane fluidity and enhancing solubility of carrier systems due to their 
satisfactory biocompatibility. ${ }^{42}$ Arsenic bioavailability is elevated by phospholipids; with the supportive agent phospholipids, arsenic can go through cell layers with high transport rate. Arsenic-induced positive correlation between Lachnospiraceae and phospholipids may be another way for arsenic impact on health.

\section{Arsenic-induced gut microbiome and its metabolic profile alterations are reversible to some extent after a recovery period}

Human hosted microbiomes can metabolize arsenic when cultured in vitro. ${ }^{43,44}$ Microbes in human gut protect their host against the arsenic toxicity. ${ }^{45}$ Thus, it is questioned, whether the gut microbiota will relieve the symptoms of arsenic poisoning or not? Our results show that the taxonomic richness did not, but the diversity significantly recovered after the 30-days recovery following the arsenic treatment. We infer that is caused by a decrease in the taxonomic evenness of the community. Arsenic may not impact all species equally, but weaken the few dominant species (i.e., the hub nodes in the free-scale network theory) who have controlled the many disadvantage species in the system. ${ }^{46}$ When the species balance is disrupted, the components and correlations in a system will lose the original orders and add the evenness. This effect lessens after a recovery period, resulting in a decrease in the taxonomic evenness of the community. Microbiomes from the As-treated groups and As-recovery groups are separated in the PCoA, which may indicate a restoration effect, but this restoration effect is not dose-depend.

At phylum level, the taxonomic assignments of Firmicutes, Proteobacteria and Bacteroidetes were closely resembled to its baseline state by 30 days after the end of arsenic treatment. Firmicutes can ferment indigestible food into substances such as short chain fatty acids (SCFA) in the intestine, and these metabolites can be absorbed through specific absorption processes and provide maximum energy to the body. ${ }^{47}$ Their restoration can effectively reduce the damage of arsenic to the body. These restorations can also be proved by the recovery of serum biochemical parameters (Additional file 1: Figure S5). However, Bacteroidetes failed to recover within 30 days in the $6.25 \mathrm{mg} / \mathrm{L}$ group, we could not determine whether it was caused by the arsenic-exposed concentration or insufficient recovery time.

Lactobacillus was identified as characteristic mircobiota after recovery. It is an antimicrobial and antioxidative probiotic strain with protective effect by promoting a stable gut microbial community. Lactobacillus strains in vitro partially revert the oxidative stress, the response of pro-inflammatory cytokines, the alterations in tight junction proteins distribution, and the cell permeability increases caused by inorganic arsenic. ${ }^{48}$ These evidences illustrate the important role of Lactobacillus to protect their host against the arsenic toxicity.

The five mainly perturbed metabolic pathways, especially glycerophospholipid metabolism were observed after arsenic treatment, which are also the predicted functional metabolic pathways affected by gut microbiota (Additional file 2: Table S8). After the 30-days recovery, the numbers of significant 
differential metabolites were less identified (Fig. 6), which indicate the perturbations of metabolic profiles have a great recovery. However, there are still four enrichment metabolic pathways were found. For example, the purine metabolism was significantly changed, number of disorders of purine metabolism may lead to immunodeficiency. ${ }^{49} \mathrm{~A}$ persistent impact of arsenic on glycerophospholipid metabolism was observed either a recovery period. Previously, the metabolism of gut microbial glycerophospholipids can promote CVD. ${ }^{5}$ These implied the lasting risk of arsenic.

Interestingly, glycerophospholipid metabolism was the significantly disturbed metabolic pathway, but it partially recovered after the 30-day recovery period. Lactobacillus was the unique representative gut species, and it has a negative correlations with $\mathrm{PE}(34: 1)$, which increased significantly after the 30-day recovery. Therefore, we infer that the therapeutic effect of Lactobacillus on arsenic exposure may be achieved by reducing excessive $\mathrm{PE}(34: 1)$ and thereby restoring the abnormal disturbance of glycerophospholipid metabolism. Lactobacillus strains may work as protective agents against the arsenic-induced health damage.

\section{Conclusions}

In this study, an integration analysis of 16S rRNA sequencing and untargeted metabolomics was performed to investigate the impact of arsenic on the gut microbiome and its metabolic profiles in rats. Arsenic significantly modified the structure and composition of gut bacteria, as well as a number of metabolites involved in the diverse metabolic pathways were observed. Furthermore, there was a strong correlation between some specific gut bacteria and the selected differential metabolites. We demonstrated the microbial dysbiosis and dysregulation of microbial metabolism might be potential early events of arsenic-induced human diseases. More importantly, arsenic-induced disruptions of gut microbiome and metabolome are reversible to some extent after a recovery period, but the exposure could affect the balance of gut microbiota with the lasting consequences. However, due to experiment condition and time limitations, the recovery period lasted only 30 days in this study. We are not sure whether the arsenic-induced effects of gut microbiota will be restored fully after a longer recovery period, or may never be completed. In summary, more in-vivo and epidemiological studies are required to explore the persistence and reversibility of arsenic-induced alterations in the gut microbiome and metabolome.

\section{Methods}

\section{Study design and sample collection}

The animal experiments were approved by the ethical committee of Institute of Urban Environment, Chinese Academy of Sciences. Eighty weaned male Wister rats from Xiamen University Laboratory Animal Centre were administrated. The whole process has followed humane care. Rats were free to access the food and water. They were housed with a cycle of $12 \mathrm{~h}$ of light and $12 \mathrm{~h}$ of darkness. 
After the acclimatization for one week, the rats (PND 23, weight $63 \pm 2 \mathrm{~g}$ ) were randomly divided into ten groups ( $\mathrm{n}=8$ per group). Two control group rats received deionized water alone, which are the 30 -days control group (PND 53) and 60-days control group (PND 83). Four group rats (As-treated groups) were treated with $0.05,0.25,1.25$ and $6.25 \mathrm{mg} / \mathrm{L}$ ATO via water drinking route for 30 days, respectively. The concentrations were selected based on environmental arsenic levels in groundwater in the heavily polluted areas, such as Bangladesh. The maximum permissible reference of arsenic in drinking water is $0.05 \mathrm{mg} / \mathrm{L}$. However, many reports indicate that the drinking water contain arsenic concentrations several times higher than the threshold in many areas of the different countries. ${ }^{50-53}$ The other four group rats (As-recovery groups) were the animals that firstly treated with the upper mentioned doses of ATO for 30days and then with the deionized water for the additional 30-days. Animals were sacrificed by decapitation immediately after the administrations. Blood was collected from rats of each group for serum biochemical parameters. Then the gut content was collected, kept in liquid nitrogen overnight, and stored under $-80^{\circ} \mathrm{C}$ prior to microbiome and metabolome analysis. In addition, rats were assessed every five days. No significant difference of physical condition, body weight, the food and water intake was observed between the control and arsenic-treated rats.

\section{Evaluation of Serum Biochemical Parameters}

The blood samples without anti-coagulants were allowed to clot by leaving it undisturbed at room temperature for $30 \mathrm{~min}$. Then samples were centrifuged at $3500 \mathrm{rpm}$ for $15 \mathrm{~min}$ at $4{ }^{\circ} \mathrm{C}$ for separation of serum. Collected serum was used for the estimation of serum TP, ALB, TBA, LDH, a-HBDH and CK-MB. These biochemical parameters analysis was detected by an BS-240VET automatic biochemical analyzer (Shenzhen Mairui Biological Medical Electronic Co., Ltd., China).

\section{Microbiome analysis}

The genomic DNA was extracted from fecal sample using the HiPure Stool DNA Kit (Magen Biotechnology Co., Ltd., China) according to the manufacturer's guidelines. The concentration and purity of genomic DNA were checked by the absorbance ratios at A260/A280 and A260/A230 using a NanoDrop ${ }^{\mathrm{TM}} 2000$ spectrophotometer (Thermo Fisher Scientific, USA). The DNA was used in the study only when the A260/A280 ratio was larger than 1.7 and the A260/A230 ratio was larger than 1.8 . The purified DNA was stored at $-80^{\circ} \mathrm{C}$ for later use.

DNA was amplified using the primers (341F: 5'-CCTACGGGNGGCWGCAG-3' and 806R: 5'GGACTACHVGGGTATCTAAT-3') with barcode directionally targeting the V3 and V4 hypervariable regions of the 16S rRNA gene on a thermocycler PCR system (ETC811, Eastwin Scientific Equipments Inc., China) (Additional file 1: Method S1). All the samples were amplified in triplicate and combined before purification. Amplicons were purified using the AMPure XP Beads (Beckman Coulter, USA), quantified using Qubit3.0 (Thermo Fisher Scientific, USA) according to the manufacturers' protocols, respectively. The sequencing libraries were constructed by the GENEDENOVO Biological Technology Co. Ltd 
(Guangzhou, China). The purified amplicons were pooled at the equimolar concentrations, and index codes were added. The libraries were sequenced with the Illumina HiSeq2500 system (Illumina, USA).

The sequence reads were generated and assigned to each sample based on their barcodes. Quality control of FASTQ files performed by FASTP (https://github.com/OpenGene/fastp). ${ }^{54}$ Then, the paired-end reads were merged by Fast Length Adjustment of SHort reads (FLASH; http://ccb.jhu.edu/software/FLASH/). ${ }^{55}$ In order to obtain the high-quality clean tags, files were qualityfiltered using Quantitative Insights Into Microbial Ecology (QIIME; http://qiime.org/). ${ }^{56}$ Chimeric sequences were detected and removed by UCHIME (http://drive5.com/uchime), ${ }^{57}$ thereby getting a set of effective tags. Effective tags were used to perform OTUs cluster and species annotation. OTU sequences were assigned with $\geq 97 \%$ similarity using UPARSE (version 9.0.2132). ${ }^{58}$ Classifications were determined by comparing sequences to the Ribosomal Database Project (RDP) classifier. ${ }^{59}$ Species diversity difference analysis was conducted by alpha diversity, including the cha01, Shannon and Simpson's diversity indices, and beta diversity in QIIME (version 1.8.0).

\section{Metabolite profiling analysis}

The metabolome was extracted from fecal sample using methanol (Additional file 1: Method S2), and a quality control (QC) sample was prepared by maxing aliquots of each sample. Metabolic profile acquisition was conducted on a Q-Exactive mass spectrometer (Thermo Scientific, USA) with electrospray ionization (ESI) source. The mass spectrometer was interfaced with a Waters ACQUITY UPLC System (Waters, Milford, MA, USA). Chromatographic separation was performed on an ACQUITY UPLC HSS T3 column $(1.8 \mu \mathrm{m}, 100 \mathrm{~mm} \times 2.1 \mathrm{~mm}$ i.d.) (Waters, Milford, MA, USA). The detail of metabolic profiling acquisition is shown in Method S3, Additional file 1.

The process of metabolomics data has been described previously. ${ }^{60}$ Then, the normalized data was submitted to SIMCA-P software (version 13.0, Umetrics, Uppsala, Sweden) for principal component analysis (PCA) and OPLS-DA model. 999-time permutation test was performed to validate the developed OPLS-DA models. Metabolic features with variable influence on projections $(\mathrm{VIP})>2$, $p$-value of the features between paired treatment-control groups $<0.05$, and fold change $(F C)>2$ were considered to be the potential biomarkers. Based on accurate mass measurement, metabolites identification with significant changes was searched against the Human Metabolome Database (HMDB;

http://www.hmdb.ca/) with a 10-ppm molecular weight tolerance. Furthermore, the UPLC/MS/MS product ion spectrum of a metabolite was matched with the MS spectra available in HMDB to confirm the identification. The identified biomarkers were subjected to MetaboAnalyst 4.0 (https://www.metaboanalyst.ca/faces/home.xhtml) for the pathway enrichment analysis and Cytoscape 3. 6.1 software for the correlation network analysis $(|r|>0.6$ and $p<0.05$, $p$-values were adjusted with FDR). ${ }^{61}$ 


\section{Microbiome and metabolome correlation analysis}

Correlations between the differential metabolites and bacterial communities were assessed by Spearman's correlation analysis with SPSS. The Rho were computed for all pairs between specific gut bacterial species and differential metabolites, including the TOP 10 differential metabolites that mostly associated with other metabolites and differential metabolites that significantly affected their metabolic pathways. P-values were adjusted with FDR. The interactions with $|r|>0.6$ and $p<0.01$ were focused on. Heat maps were generated using $\mathrm{R}$ to visualize the correlation between microbiota and metabolites.

\section{Statistical analysis}

Bioinformatic analysis was performed using Omicsmart, a dynamic real-time interactive online platform for data analysis (http://www.omicsmart.com). The statistical analysis was performed using SPSS software (version 18.0) and the data were expressed as mean \pm S.D. All the data were analyzed using Mann-Whitney $U$ test, and $p<0.05$ was statistically significant.

\section{Abbreviations}

ATO: arsenic trioxide; PCoA: principal coordinates analysis; OTU: operational taxonomic units; LEfSe: linear discriminant analysis integrated with effect size; OPLS-DA: orthogonal partial least-squares discriminant analysis; 4-HPPA: 4-hydroxyphenylpyruvic acid; HPG: hydroxyphenylacetylglycine; VG: vinylacetylglycine; GNP: glucosamine 6-phosphate; Laminine: N6,N6,N6-trimethyl-L-lysine; MelmAA: methylimidazoleacetic acid; MHPG: 3-methoxy-4-hydroxyphenylglycolaldehyde; 4,6-DHQ: 4,6dihydroxyquinoline; MIA: 5-methoxyindoleacetate; AFMK: acetyl-N-formyl-5-methoxykynurenamine; GIcNAc-6-P: N-acetyl-D-glucosamine 6-phosphate; GlcNAcb: N-acetyl-b-glucosaminylamine; Glc-1P: glucose 1-phosphate; PEA-G: phenethylamine glucuronide; DMPQ: demethylphylloquinone; G3P: glycerol 3-phosphate; T: testosterone; 3b-OH- $\triangle 5$ : 3b-hydroxy-5-cholenoic acid; 17-OHP: 17-hydroxyprogesterone; 2-MBC: 2-methylbutyroylcarnitine; 7-KDC: 7-ketodeoxycholic acid; CS: cholesterol sulfate; 7a-HCO: 7ahydroxy-cholestene-3-one; N: nandrolone; 11b-OHP: 11b-hydroxyprogesterone; TC: tetradecanoylcarnitine; 4,8 DNC: 4,8 dimethylnonanoyl carnitine; DPA: docosapentaenoic acid (22n-3); STA: stearidonic acid; 17a,21-DDP: 17alpha,21-dihydroxypregnenolone; PC: phosphatidylethanolamine; PE: phosphatidylethanolamine; LysoPC: lysophosphatidylcholine; LysoPE: lysophosphatidylethanolamine; MG: monoglyceride; 5,8,11-ET: 5,8,11-eicosatrienoic acid; 4a-F-4b-methylzymosterol: 4alpha-formyl-4betamethyl-5alpha-cholesta-8,24-dien-3beta-ol; 11'-CGT3: 11'-carboxy-gamma-tocotrienol; CEA: cervonoyl ethanolamide; 16a-HEA-3S: 16alpha-hydroxydehydroepiandrosterone 3-sulfate; 7a-H-30A: 7alphahydroxy-3-oxochol-4-en-24-oic acid; DI: deoxyinosine; DG: deoxyguanosine; PRA: 5-phosphoribosylamine; MTA: 5'-methylthioadenosine; Gin-Lys: epsilon-(gamma-glutamyl)-lysine; LP: leucylproline; $\quad$ Ala-Leu: alanylleucine; Ala-Tyr: alanyltyrosine; Arg-Gly: arginylglycine; Glu-Val: glutaminylvaline; SL: serylleucine; STP: seryltryptophan; GPI: glycosylphosphatidylinositol; ASD: autism spectrum disorder; TP: total protein; ALB: albumin; TBA: total bile acid; LDH: lactic dehydrogenase; a-HBDH: alpha-hydroxybutyric dehydrogenase; CK-MB: creatine kinase MB isoenzyme; CVD: cardiovascular disease; MI: myocardial infarction; SCFA: short chain fatty acids; FLASH: Fast Length Adjustment of SHort reads; QIIME: 
Quantitative Insights Into Microbial Ecology; RDP: Ribosomal Database Project; QC: quality control; ESI: electrospray ionization; PCA: principal component analysis; VIP: variable influence on projections; FC: fold change; HMDB: Human Metabolome Database.

\section{Declarations}

\section{Ethics approval and consent to participate}

The study was carried out in accordance with Laboratory Animal-Guideline for Ethical Review of Animal Welfare (GB/T 35892-2018). The animal experiments were approved by the ethical committee of Institute of Urban Environment, Chinese Academy of Sciences.

\section{Consent for publication}

Not applicable.

\section{Availability of data and materials}

Parts of data generated or analysed during this study are included in the supplementary information files of this article. Other datasets are available from the corresponding author on reasonable request.

\section{Competing interests}

The authors declare that they have no competing interests.

\section{Funding}

National Natural Science Foundation of China (No. 21677141, 21777157), Fundamental Research Funds for the Central Universities (No. 20720190074), Natural Science Foundation of Fujian Province (No. 2018J01021).

\section{Acknowledgments}

The first author acknowledges the financial support from the DAAD-UCAS. We also acknowledge the assistance of Yan Wu in the collection of the rat gut content samples.

\section{Authors' information}

\section{Affiliations}

Key Lab of Urban Environment and Health, Institute of Urban Environment, Chinese Academy of Sciences, Xiamen, China

Xiaoyan Du, Jie Zhang, Qingyu Huang, Meiping Tian, Xi Zhang, Bingru Nan \& Heqing Shen 
State Key Laboratory of Molecular Vaccinology and Molecular Diagnostics, School of Public Health, Xiamen University, Xiamen, China

Jie Zhang \& Heqing Shen

University of Chinese Academy of Sciences, Beijing, China

Xiaoyan Du, Xi Zhang \& Bingru Nan

Helmholtz Center Munich-German Research Center for Environmental Health, Molecular EXposomics, Neuherberg, Germany

Karl-Werner Schramm

Department für Biowissenschaftliche Grundlagen, Technische Universität München, Freising, Germany Karl-Werner Schramm

\section{Contributions}

$\mathrm{XZ}$ and $\mathrm{BN}$ performed the experiments and collected the data. XD, JZ, KWS, QH, MT contributed to the data analysis and interpretation. JZ, and HS contributed to the funding acquisition. All authors read and approved the final manuscript.

\section{Corresponding authors}

Jie Zhang at State Key Laboratory of Molecular Vaccinology and Molecular Diagnostics, School of Public Health, Xiamen University, Xiamen, China. Tel/Fax: 86-592-2880677; E-mail: jie.zhang@xmu.edu.cn

or

Heqing Shen at Key Lab of Urban Environment and Health, Institute of Urban Environment, Xiamen, China 361021. Tel/Fax: 86-592-6190771; E-mail: hqshen@iue.ac.cn.

\section{References}

1. Nordstrom DK. Worldwide occurrences of arsenic in ground water. Science. 2002;296(5576):21432145.

2. Kapaj S, Peterson H, Liber K, Bhattacharya P. Human health effects from chronic arsenic poisoning-a review. J Environ Sci Health Part A Toxic/Hazard Subst Environ Eng. 2006;41(10):2399-2428.

3. Masotti A, Sacco LD, Bottazzo GF, Sturchio E. Risk assessment of inorganic arsenic pollution on human health. Environ Pollut. 2009;157(6):1770-1772.

4. Caesar R, Fåk F, Bäckhed F. Effects of gut microbiota on obesity and atherosclerosis via modulation of inflammation and lipid metabolism. J Intern Med. 2010;268(4):320-328. 
5. Wang Z, Klipfell E, Bennett BJ, Koeth R, Levison BS, DuGar B, Feldstein AE, Britt EB, Fu X, Chung YM, et al. Gut flora metabolism of phosphatidylcholine promotes cardiovascular disease. Nature. 2011;472(7341):57-63.

6. Lee WJ, Hase K. Gut microbiota-generated metabolites in animal health and disease. Nat Chem Biol. 2014;10(6):416-424.

7. Pickard JM, Zeng MY, Caruso R, Núñez G. Gut microbiota: role in pathogen colonization, immune responses, and infammatory disease. Immunol Rev. 2017;279(1):70-89.

8. Schroeder BO, Bäckhed F. Signals from the gut microbiota to distant organs in physiology and disease. Nat Med. 2016;22(10):1079-1089.

9. Nicholson JK, Holmes E, Kinross J, Burcelin R, Gibson G, Jia W, Pettersson S. Host-gut microbiota metabolic interactions. Science. 2012;336(6086):1262-1267.

10. Magnúsdóttir S, Heinken A, Kutt L, Ravcheev DA, Bauer E, Noronha A, Greenhalgh K, Jäger C, Baginska J, Wilmes P, et al. Generation of genome-scale metabolic reconstructions for 773 members of the human gut microbiota. Nat Biotechnol. 2017;35(1):81-89.

11. Dheer R, Patterson J, Dudash M, Stachler EN, Bibby KJ, Stolz DB, Shiva S, Wang Z, Hazen SL, Barchowsky A, et al. Arsenic induces structural and compositional colonic microbiome change and promotes host nitrogen and amino acid metabolism. Toxicol Appl Pharmacol. 2015;289(3):397-408.

12. Lu K, Abo RP, Schlieper KA, Graffam ME, Levine S, Wishnok JS, Swenberg JA, Tannenbaum SR, Fox JG. Arsenic exposure perturbs the gut microbiome and its metabolic profile in mice: an integrated metagenomics and metabolomics analysis. Environ Health Perspect. 2014;122(3):284-291.

13. Chi L, Bian X, Gao B, Ru H, Tu P, Lu K. Sex-specific effects of arsenic exposure on the trajectory and function of the gut microbiome. Chem Res Toxicol. 2016;29(6):949-951.

14. Cani PD, Delzenne NM. The role of the gut microbiota in energy metabolism and metabolic disease. Curr Pharm Des. 2009;15(13):1546-1558.

15. Tremaroli V, Bäckhed F. Functional interactions between the gut microbiota and host metabolism. Nature. 2012;489(7415):242-249.

16. Walter J. Murine gut microbiota-diet trumps genes. Cell Host Microbe. 2015;17(1):3-5.

17. David LA, Maurice CF, Carmody RN, Gootenberg DB, Button JE, Wolfe BE, Ling AV, Devlin AS, Varma Y, Fischbach MA, et al. Diet rapidly and reproducibly alters the human gut microbiome. Nature. 2014;505:559-563.

18. Turnbaugh PJ, Bäckhed F, Fulton L, Gordon JI. Diet-induced obesity is linked to marked but reversible alterations in the mouse distal gut microbiome. Cell Host Microbe. 2008;3(4):213-223.

19. Theriot CM, Koenigsknecht JM, Carlson Jr PE, Hatton GE, Nelson AM, Li B, Huffnagle GB, Li JZ, Young VB. Antibiotic-induced shifts in the mouse gut microbiome and metabolome increase susceptibility to Clostridium difficile infection. Nat Commun. 2014;5:3114.

20. Jeffery IB, Lynch DB, O'Toole PW. Composition and temporal stability of the gut microbiota in older persons. ISME J. 2016;10:170-182. 
21. Robinson CJ, Bohannan BJM, Young VB. From structure to function: the ecology of host-associated microbial communities. Microbiol Mol Biol Rev. 2010;74(3):453-476.

22. Zhai Q, Cen S, Jiang J, Zhao J, Zhang H, Chen W. Disturbance of trace element and gut microbiota profiles as indicators of autism spectrum disorder: a pilot study of Chinese children. Environ Res. 2019;171:501-509.

23. Finegold SM, Dowd SE, Gontcharova V, Liu C, Henley KE, Wolcott RD, Youn E, Summanen PH, Granpeesheh D, Dixon D, et al. Pyrosequencing study of fecal microflora of autistic and control children. Anaerobe. 2010;16(4):444-453.

24. Liu J, Waalkes MP. Liver is a target of arsenic carcinogenesis. Toxicol Sci. 2008;105(1):24-32.

25. Hsu LI, Wang YH, Hsieh FI, Yang TY, Jeng RWJ, Liu CT, Chen CL, Hsu KH, Chiou HY, Wu MM, et al. Effects of arsenic in drinking water on risk of hepatitis or cirrhosis in persons with and without chronic viral hepatitis. Clin Gastroenterol Hepatol. 2016;14(9):1347-1355.

26. Tilg H, Cani PD, Mayer EA. Gut microbiome and liver diseases. Gut. 2016;65:2035-2044.

27. Zhu L, Baker SS, Gill C, Liu W, Alkhouri R, Baker RD, Gill SR. Characterization of gut microbiomes in nonalcoholic steatohepatitis (NASH) patients: a connection between endogenous alcohol and NASH. Hepatology. 2013;57:601-609.

28. Chen Y, Yang F, Lu H, Wang B, Chen Y, Lei D, Wang Y, Zhu B, Li L. Characterization of fecal microbial communities in patients with liver cirrhosis. Hepatology. 2011;54:562-572.

29. Qin N, Yang F, Li A, Prifti E, Chen Y, Shao L, Guo J, Chatelier EL, Yao J, Wu L, et al. Alterations of the human gut microbiome in liver cirrhosis. Nature. 2014;513:59-64.

30. Marco SZ, Debora C, Pietro C, Rocco A, Nardone OM, Marrone G, Gasbarrini A, Grieco A, Nardone G, Miele $\mathrm{L}$. The metabolic role of gut microbiota in the development of nonalcoholic fatty liver disease and cardiovascular disease. Int J Mol Sci. 2016;17(8):1225.

31. Neves AL, Coelho J, Couto L, Leite-Moreira A, RonconAlbuquerque R Jr. Metabolic endotoxemia: a molecular link between obesity and cardiovascular risk. J Mol Endocrinol. 2013;51(2):R51-R64.

32. Sagar S, Liu PP, Cooper LT. Myocarditis. Lancet. 2012;379(9817)738-747.

33. Monrad M, Ersbøll AK, Sørensen M, Baastrup R, Hansen B, Gammelmark A, Tjønneland A, Overvad K, Raaschou-Nielsen O. Low-level arsenic in drinking water and risk of incident myocardial infarction: A cohort study. Environ Res. 2017;154:318-324.

34. Lam V, Su J, Koprowski S, Hsu A, Tweddell JS, Rafiee P, Gross GJ, Salzman NH, Baker JE. Intestinal microbiota determine severity of myocardial infarction in rats. FASEB J. 2012;26:1727-35.

35. Zhou X, Li J, Guo J, Geng B, Ji W, Zhao Q, Li J, Liu X, Liu J, Guo Z, et al. Gut-dependent microbial translocation induces inflammation and cardiovascular events after ST-elevation myocardial infarction. Microbiome. 2018;6:66.

36. Alhmoud T, Kumar A, Lo CC, Al-Sadi R, Clegg S, Alomari I, Zmeili T, Gleasne CD, Mcmurry K, Dichosa AEK, et al. Investigating intestinal permeability and gut microbiota roles in acute coronary syndrome patients. Human Microbiome Journal. 2019;13:100059. 
37. Zhu W, Gregory JC, Org E, Buffa JA, Gupta N, Wang Z, Li L, Fu X, Wu Y, Mehrabian M, et al. Gut microbial metabolite TMAO enhances platelet hyperreactivity and thrombosis risk. Cell. 2016;165(1):111-124.

38. Hu XF, Zhang WY, Wen Q, Chen WJ, Wang ZM, Chen J, Zhu F, Liu K, Cheng LX, Yang J, et al. Fecal microbiota transplantation alleviates myocardial damage in myocarditis by restoring the microbiota composition. Pharmacological Research. 2019;139:412-421.

39. Yin J, Liao SX, He Y, Wang S, Xia GH, Liu FT, Zhu JJ, You C, Chen Q, Zhou L, et al. Dysbiosis of gut microbiota with reduced trimethylamine-n-oxide level in patients with large-artery atherosclerotic stroke or transient ischemic attack. J Am Heart Assoc. 2015;4(11):e002699.

40. Koren O, Spor A, Felin J, Fåk F, Stombaugh J, Tremaroli V, Behre CJ, Knight R, Fagerberg B, Ley RE, et al. Human oral, gut, and plaque microbiota in patients with atherosclerosis. PNAS. 2011;108:45924598.

41. Gould RG. Lipid metabolism and atherosclerosis. Am J Med. 1951;11(2):209-227.

42. Mendes AC, Gorzelanny C, Halter N, Schneider SW, Chronakis IS. Hybrid electrospun chitosanphospholipids nanofibers for transdermal drug delivery. Int J Pharm. 2016;510:48-56.

43. Coryell M, McAlpine M, Pinkham NV, McDermott TR, Walk ST. The gut microbiome is required for full protection against acute arsenic toxicity in mouse models. Nat Commun. 2018;9:5424.

44. Van de Wiele T, Gallawa CM, Kubachk KM, Creed JT, Basta N, Dayton EA, Whitacre S, Laing GD, Bradham K. Arsenic metabolism by human gut microbiota upon in vitro digestion of contaminated soils. Environ. Health Perspect. 2010;118(7):1004-1009.

45. Coryell M, McAlpine M, Pinkham NV, McDermott TR, Walk ST. The gut microbiome is required for full protection against acute arsenic toxicity in mouse models. Nat Commun. 2018;9:5424.

46. Barabási AL, Bonabeau E. Scale-free networks. Sci Am, 2003;288(5):60-69.

47. Jumpertz R, Le DS, Turnbaugh PJ, Trinidad C, Bogardus C, Gordon JI, Krakoff J. Energy-balance studies reveal associations between gut microbes, caloric load, and nutrient absorption in humans. Am J Clin Nutr. 2011;94(1):58-65.

48. Gareau M, Sherman P, Walker W. Probiotics and the gut microbiota in intestinal health and disease. Nat Rev Gastroenterol Hepatol. 2010;7:503-514.

49. Madsen KL. Enhancement of epithelial barrier function by probiotics. J Epithel Biol Pharmacol. 2012;5(1):55-59.

50. Anawar HM, Akai J, Mostofa KMG, Safiullah S, Tareq SM. Arsenic poisoning in groundwater: Health risk and geochemical sources in Bangladesh. Environ Int. 2002;27(7):597-604.

51. Karim MM. Arsenic in groundwater and health problems in Bangladesh. Water Res. 2000;34(1):304310.

52. Armienta MA, Segovia N. Arsenic and fluoride in the groundwater of Mexico. Environ Geochem Health. 2008;30(4):345-353. 
53. Wang JS, Wai CM. Arsenic in drinking water-a global environmental problem. J Chem Educ. 2004;81(2):207-213.

54. Chen S, Zhou Y, Chen Y, Gu J. fastp: an ultra-fast all-in-one FASTQ preprocessor. Bioinformatics. 2018;34(17):884-890.

55. Magoč T, Salzberg SL. FLASH: fast length adjustment of short reads to improve genome assemblies. Bioinformatics. 2011;27(21):2957-2963.

56. Caporaso JG, Kuczynski J, Stombaugh J, Bittinger K, Bushman FD, Costello EK, Fierer N, Peña AG, Goodrich JK, Gordon JI, et al. QIIME allows analysis of high-throughput community sequencing data. Nat Methods. 2010;7(5):335-336.

57. Bokulich NA, Subramanian S, Faith JJ, Gevers D, Gordon JI, Knight R, Mills DA, Caporaso JG. Qualityfiltering vastly improves diversity estimates from Illumina amplicon sequencing. Nat Methods. 2013;10(1):57-59.

58. Edgar RC. UPARSE: highly accurate OTU sequences from microbial amplicon reads. Nat Methods. 2013;10(10):996-998.

59. Wang Q, Garrity GM, Tiedje JM, Cole JR. Naive Bayesian classifier for rapid assignment of rRNA sequences into the new bacterial taxonomy. Appl Environ Microbiol. 2007;73(16):5261-5267.

60. Wang YX, Wu Y, Chen HG, Duan P, Wang L, Shen HQ, Lu WQ, Sun B,Wang Q, Zhang B, et al. Seminal plasma metabolome in relation to semen quality and urinary phthalate metabolites among Chinese adult men. Environ Int. 2019;129:354-363.

61. Shannon P, Markiel A, Ozier O, Baliga NS, Wang JT, Ramage D, Amin N, Schwikowski B, Ideker T. Cytoscape: a software environment for integrated models of biomolecular interaction networks. Genome Res. 2003;13(11):2498-2504.

\section{Additional Files}

Additional file 1: Result S1. Sampling depth; Result S2. Potential functional impact of As-treated and Asrecovery changes in the gut microbiome; Figure S1. Rarefaction curve evaluating the relative bacterial richness to determine whether further sequencing would identify additional OTUs; Figure S2. Shared microbial OTUs; Figure S3. Important discriminatory metabolites identified; Figure S4. Number of upregulated and down-regulated differential metabolites in different groups; Figure S5. The changes of serum biochemical parameters. Method S1. PCR reactions; Method S2. Metabolite extraction; Method S3. Acquisition of metabolic profiles.

Additional file 2: Table S1. Significance differences of species diversity indexes; Table S2. The percentage of bacteria at the phylum level; Table S3. Significance of dominant bacterial components differences; Table S4. The lists of specific OTUs; Table S5. Heatmap analysis for 80 differential metabolites; Table S6. The TOP 10 differential metabolites most associated with other metabolites; Table S7. The metabolites were related to the significantly affected metabolic pathways; Table S8. The predicted functional metabolic pathways. 


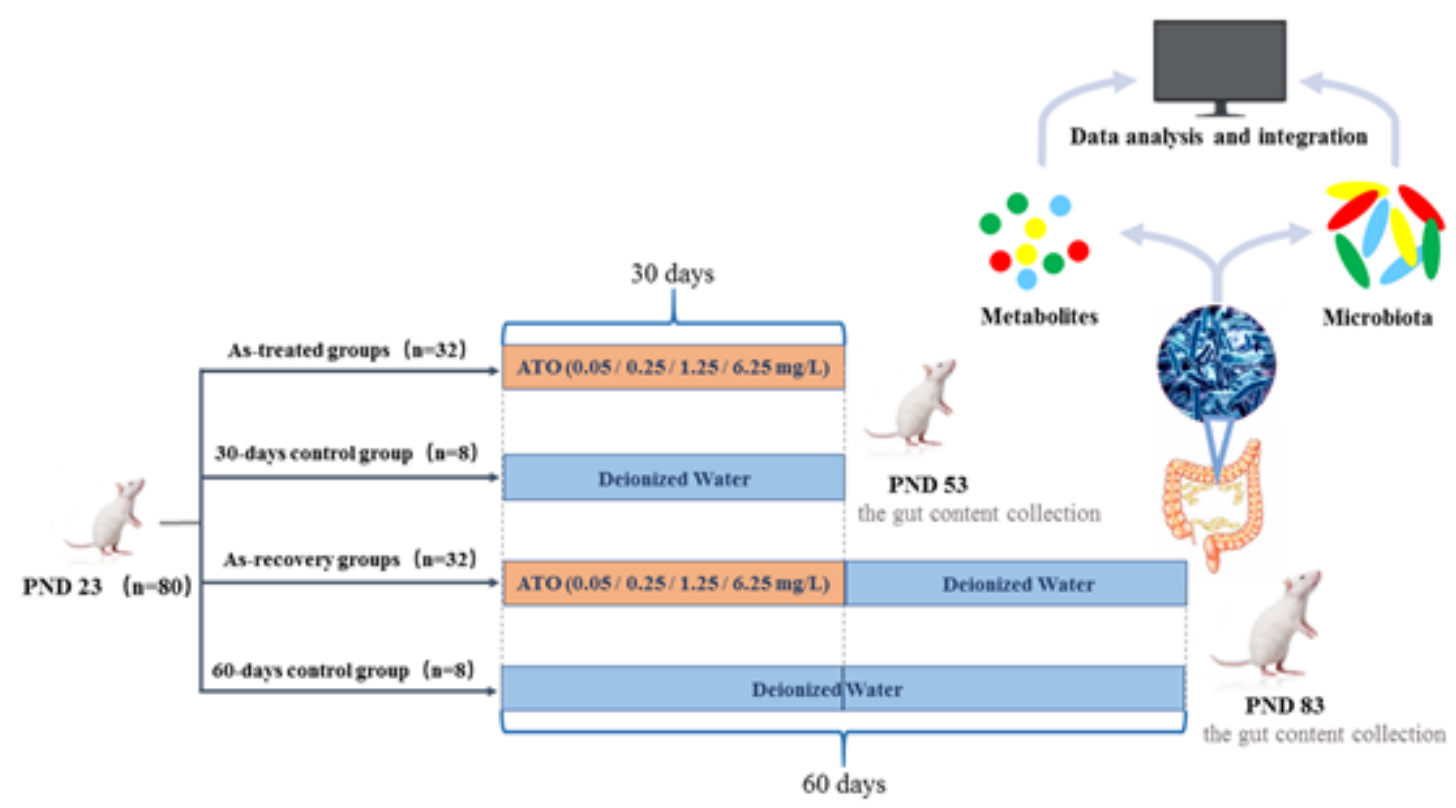

Figure 1

Experiment diagram. Eighty weaned male Wister rats (PND 23) were randomly divided into ten groups ( $\mathrm{n}=8$ per group). The rats in 30-days control group (PND 53) and 60-days control group (PND 83) received deionized water alone. Four group rats (As-treated groups) were treated with $0.05,0.25,1.25$ and 6.25 $\mathrm{mg} / \mathrm{L}$ arsenic trioxide (ATO) via water drinking route for 30 days, respectively. The other four group rats (As-recovery groups) were the animals that firstly treated with the upper mentioned doses of ATO for 30days and then with the deionized water for the additional 30-days.

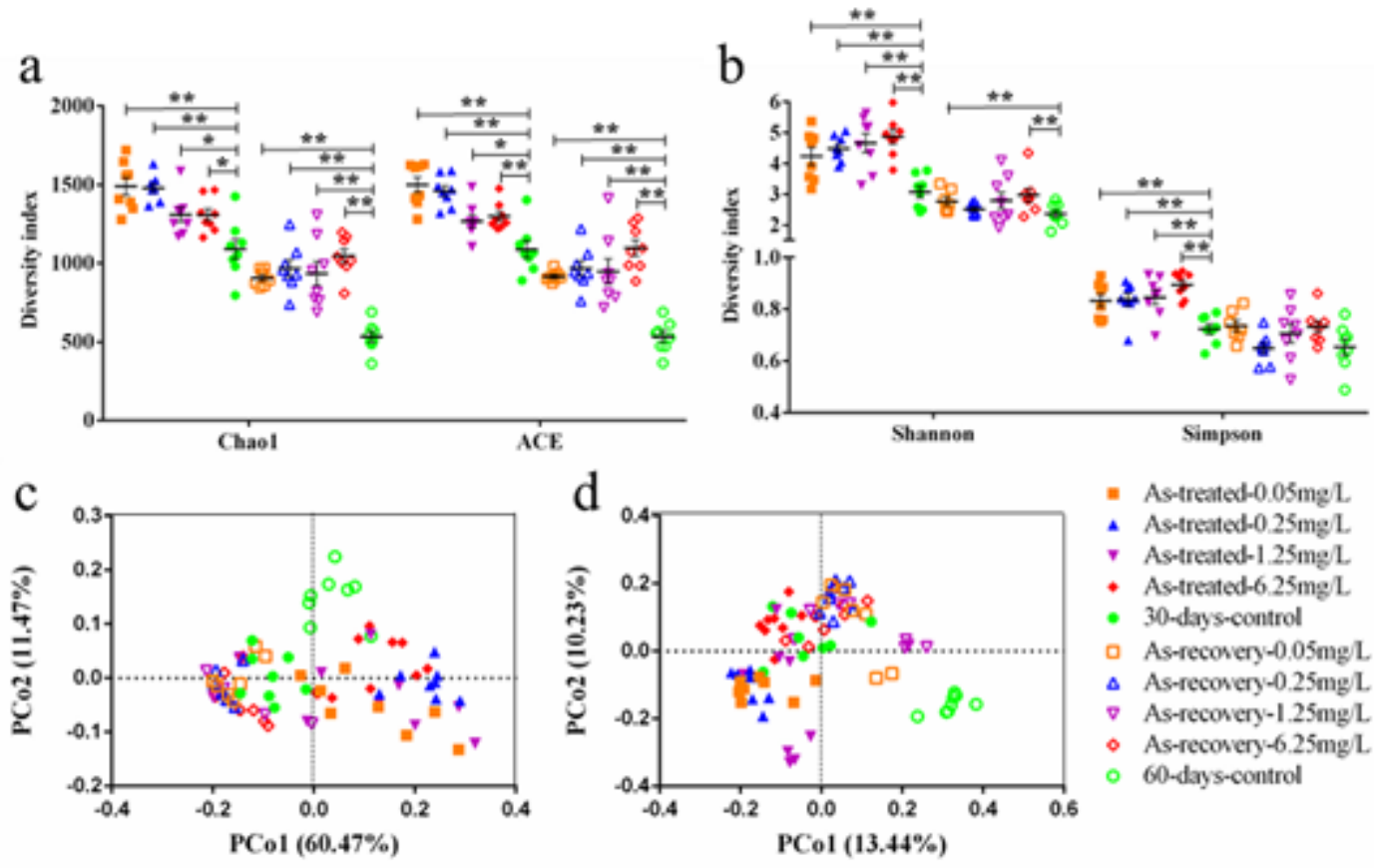

Figure 2 
Gut microbiome diversity and structure analysis. Species diversity differences were estimated by the Chao1 and ACE (a), Shannon and Simpson (b). PCoA plot base of the relative abundance of the operational taxonomic units (OTUs) (97\% similarity level) showing bacterial structural cluster. (c) Weighted unifrac PCoA plots; (d) Unweighted unifrac PCoA plots. ${ }^{*} p<0.05 ;{ }^{*} p<0.01$.

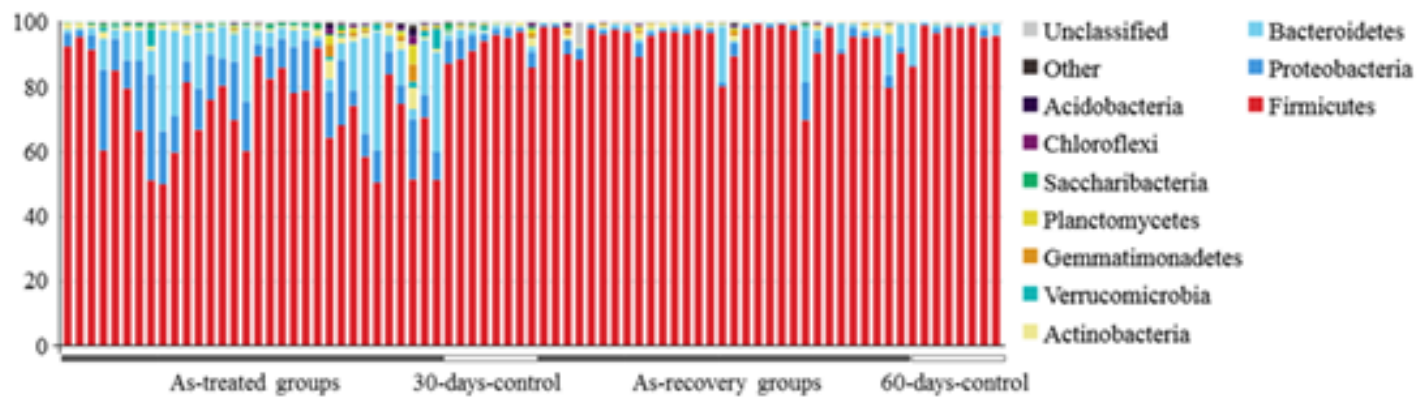

\section{Figure 3}

The gut microbiome composition profiles at the phylum level. Firmicutes, Proteobacteria and Bacteroidetes were predominant in the gut microbiota of rats at the phylum level.

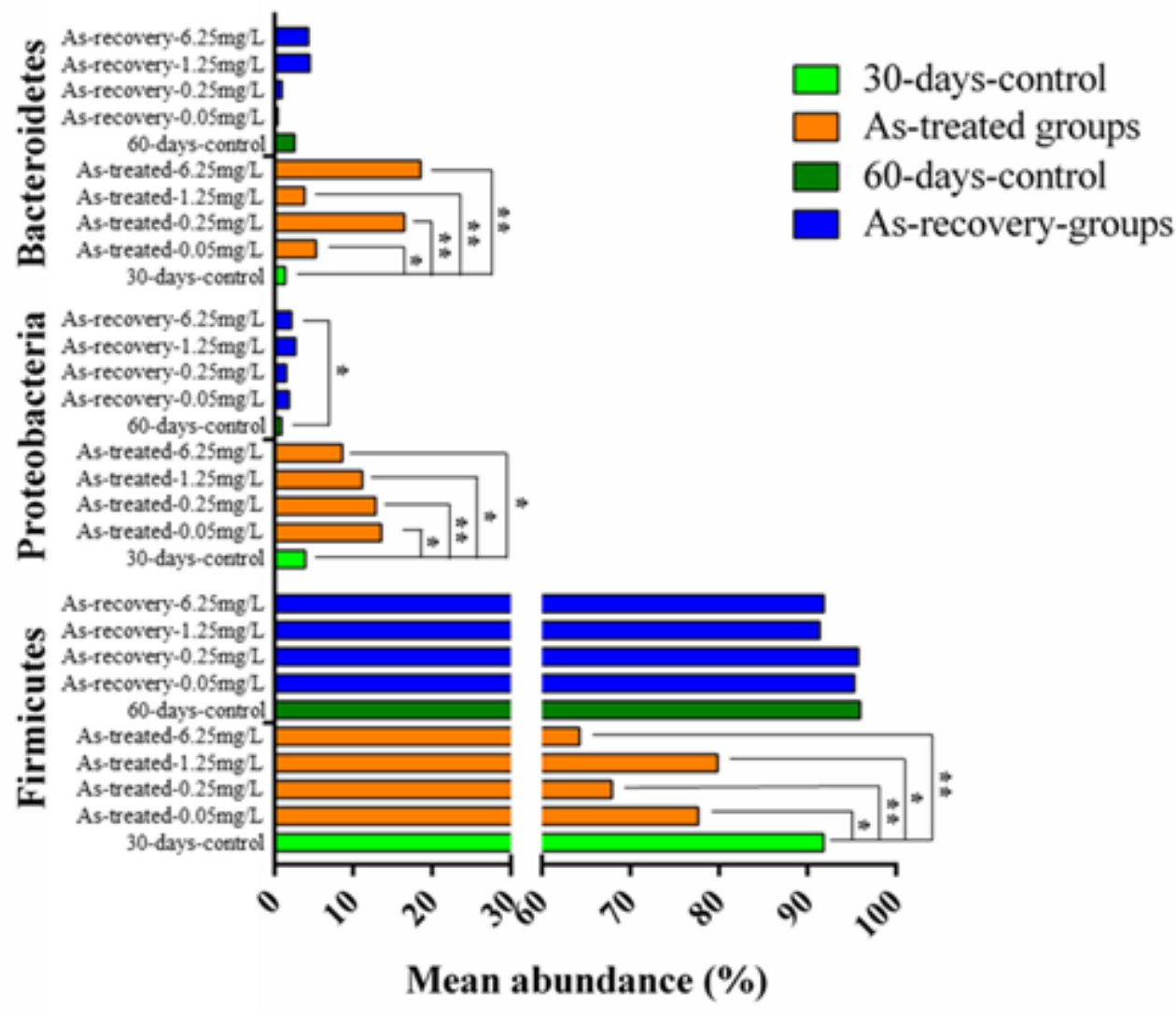

Figure 4

Welch's t-test results for evaluating significance of the relative abundance for the three predominant microbiotas at the phylum level. Firmicutes decreased significantly, whereas Proteobacteria and 
Bacteroidetes increased significantly after arsenic exposure. The relative proportions of the three phyla in As-recovery groups were similar to those in 60-days control group.

a

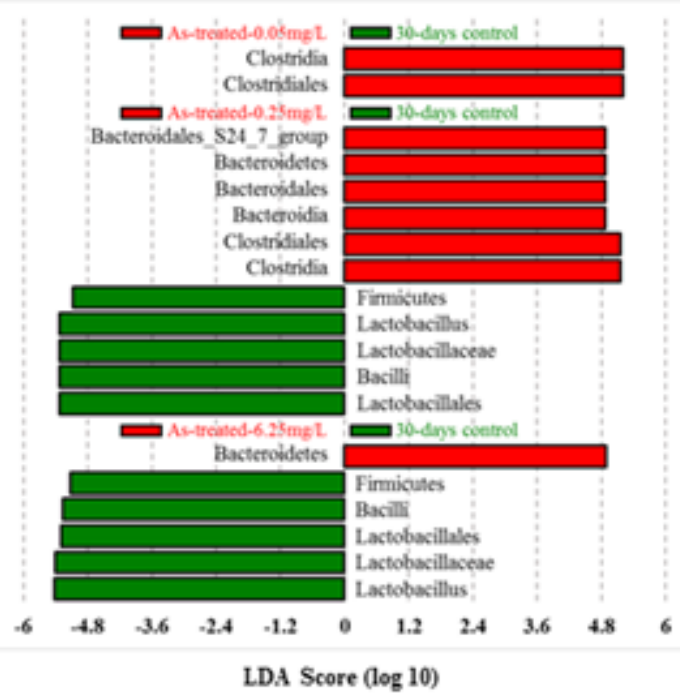

b

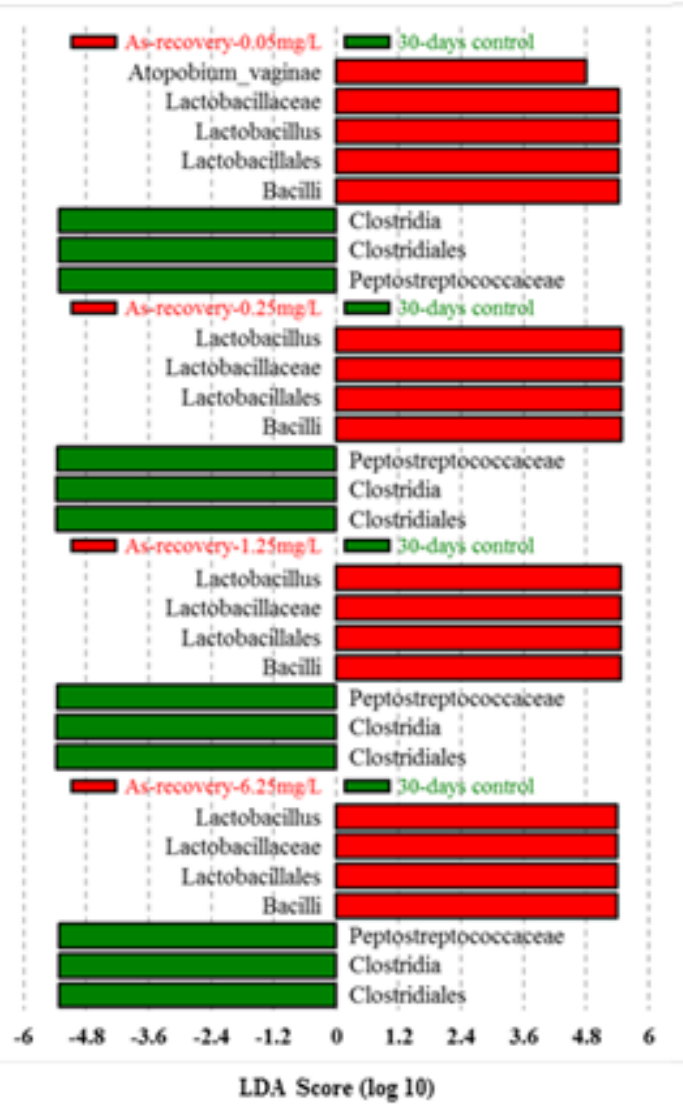

Figure 5

Linear discriminant analysis (LDA) integrated with effect size (LEfSe). The abundance discriminated and administration-specific OTUs in the As-treated groups (a) and the As-recovery groups (b). 


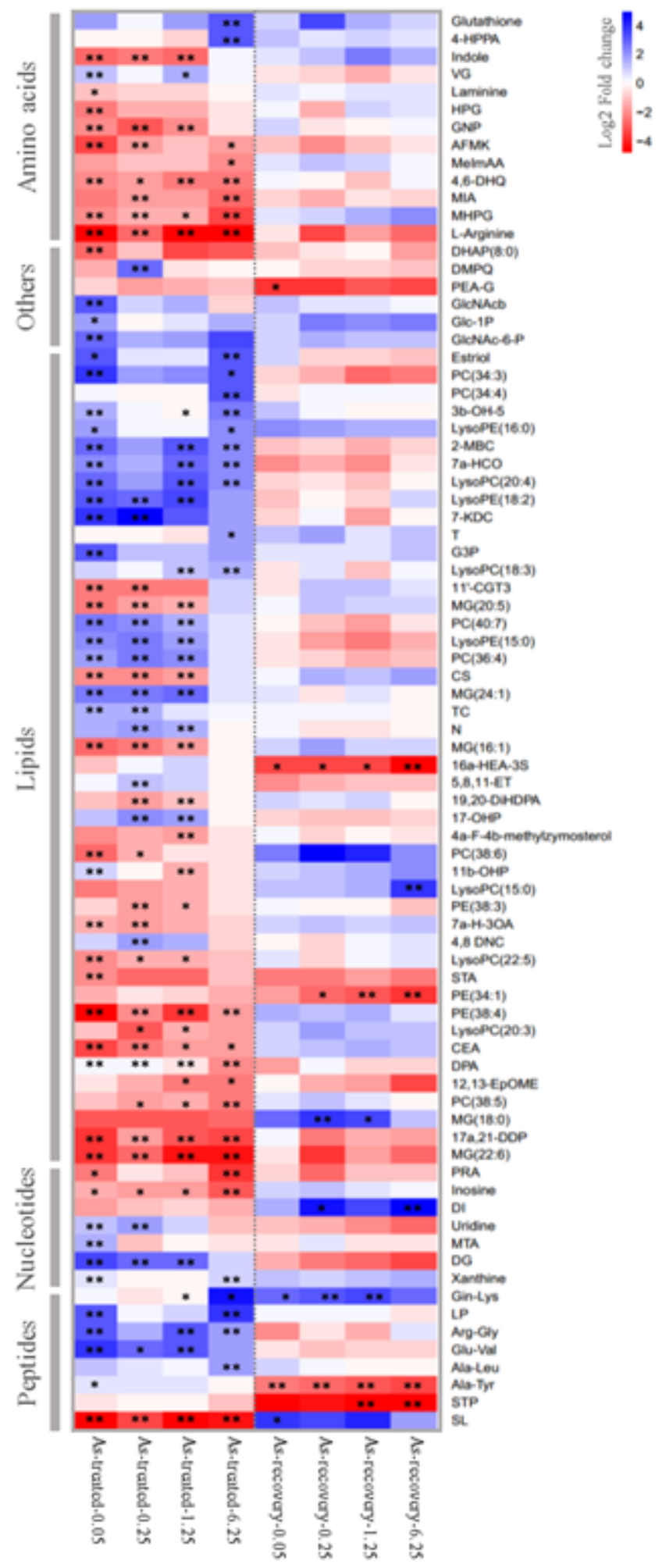

Figure 6

Heatmap showing the differentially accumulated and significantly changed metabolites in the As-treated groups and As-recovery groups. ${ }^{\star} p<0.05 ;{ }^{* *} p<0.01$. 


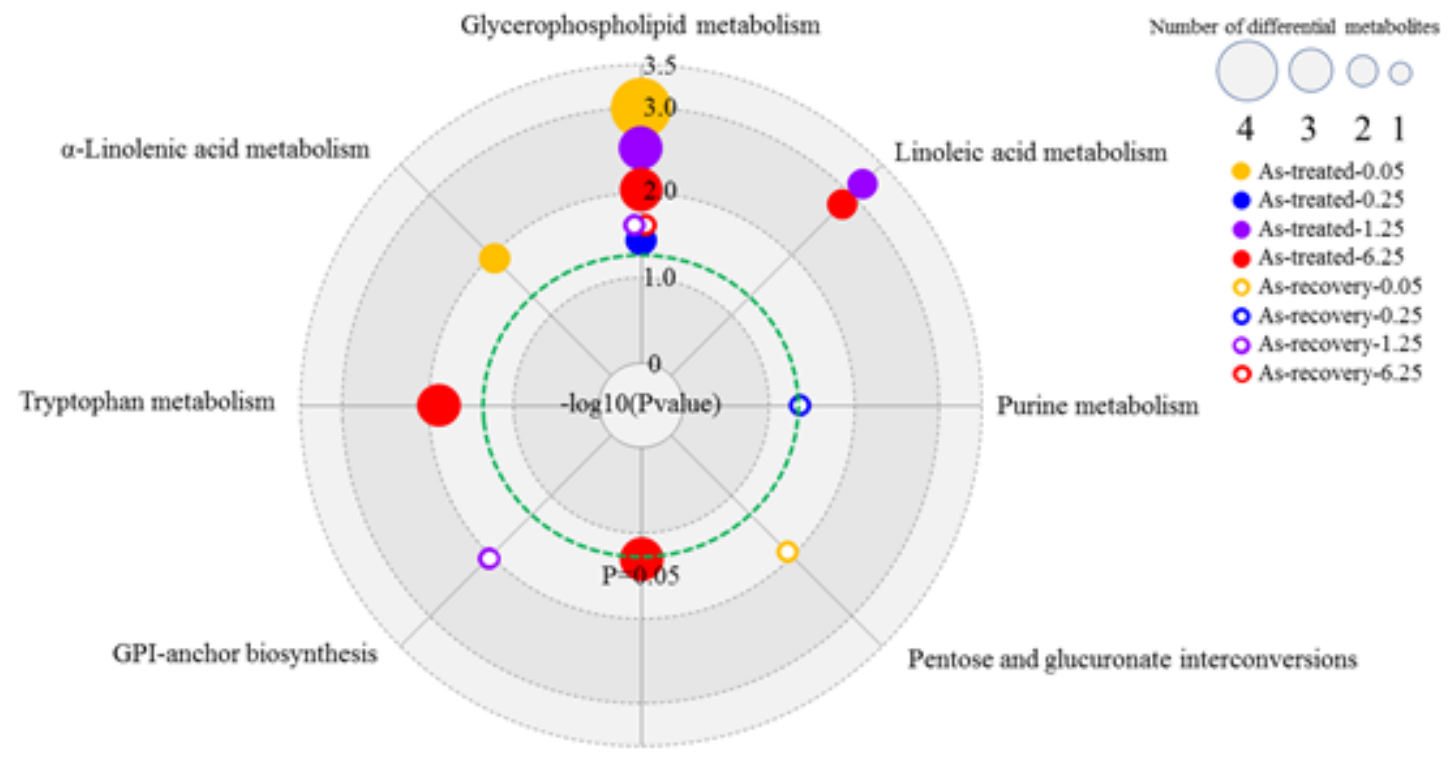

Phenylalanine, tyrosine and tryptophan biosynthesis

\section{Figure 7}

Radar map of gut microbial metabolic pathway-enrichment analysis of differential metabolites in the Astreated groups and As-recovery groups.

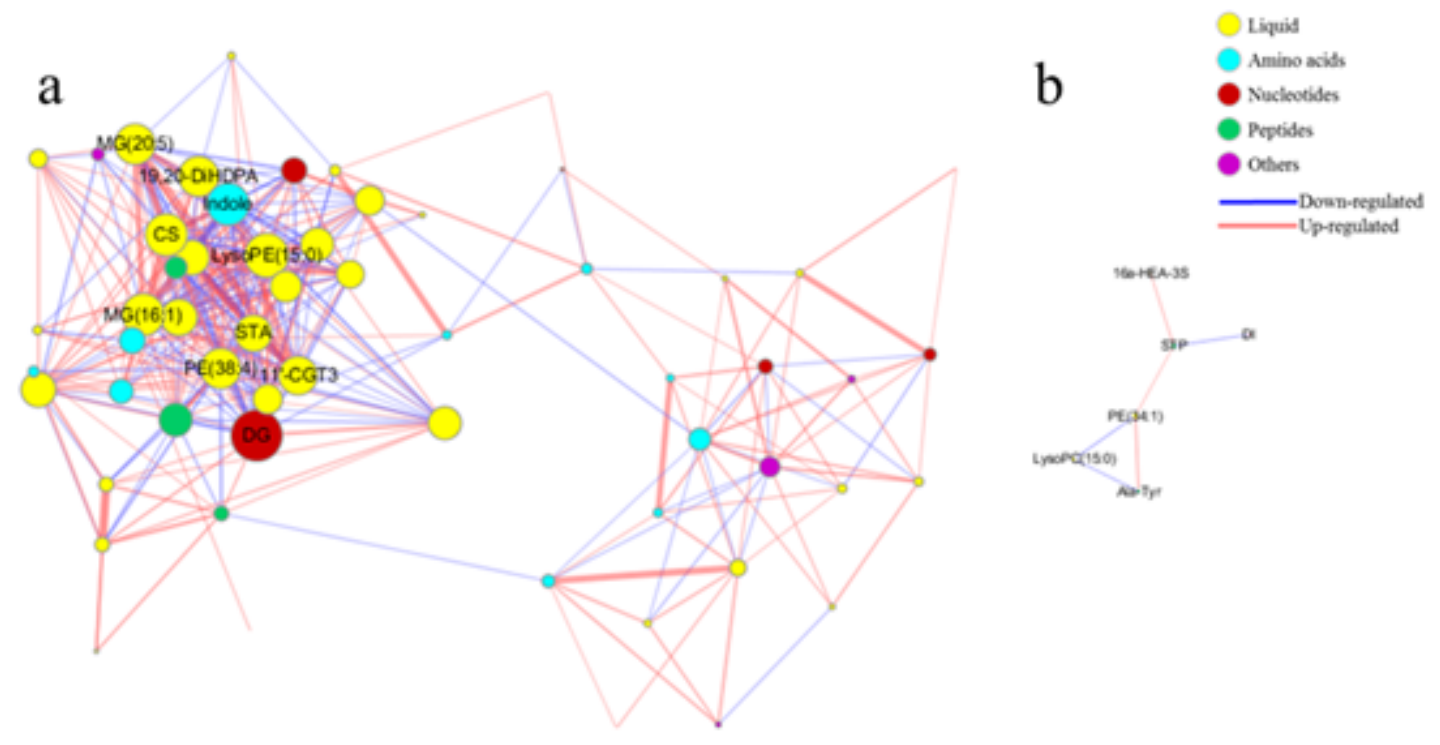

\section{Figure 8}

Metabolic correlation networks of metabolic correlation network of the As-treated groups (a) and the Asrecovery groups (b). TOP 10 differential metabolites that mostly associated with the other ones were tagged. 


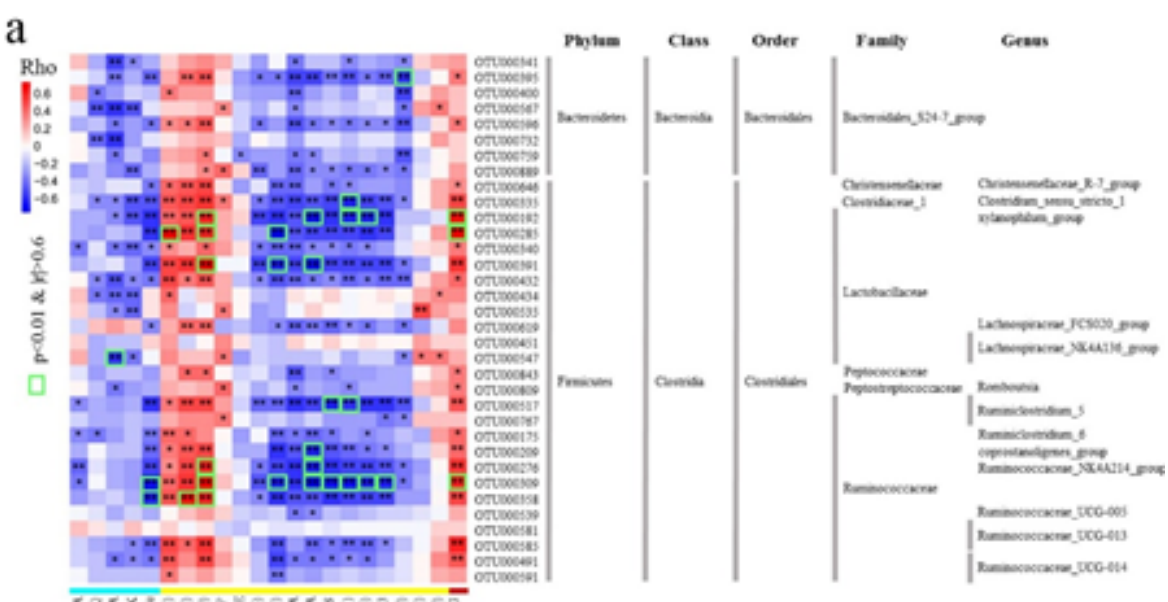

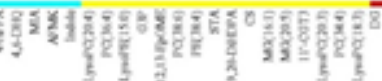

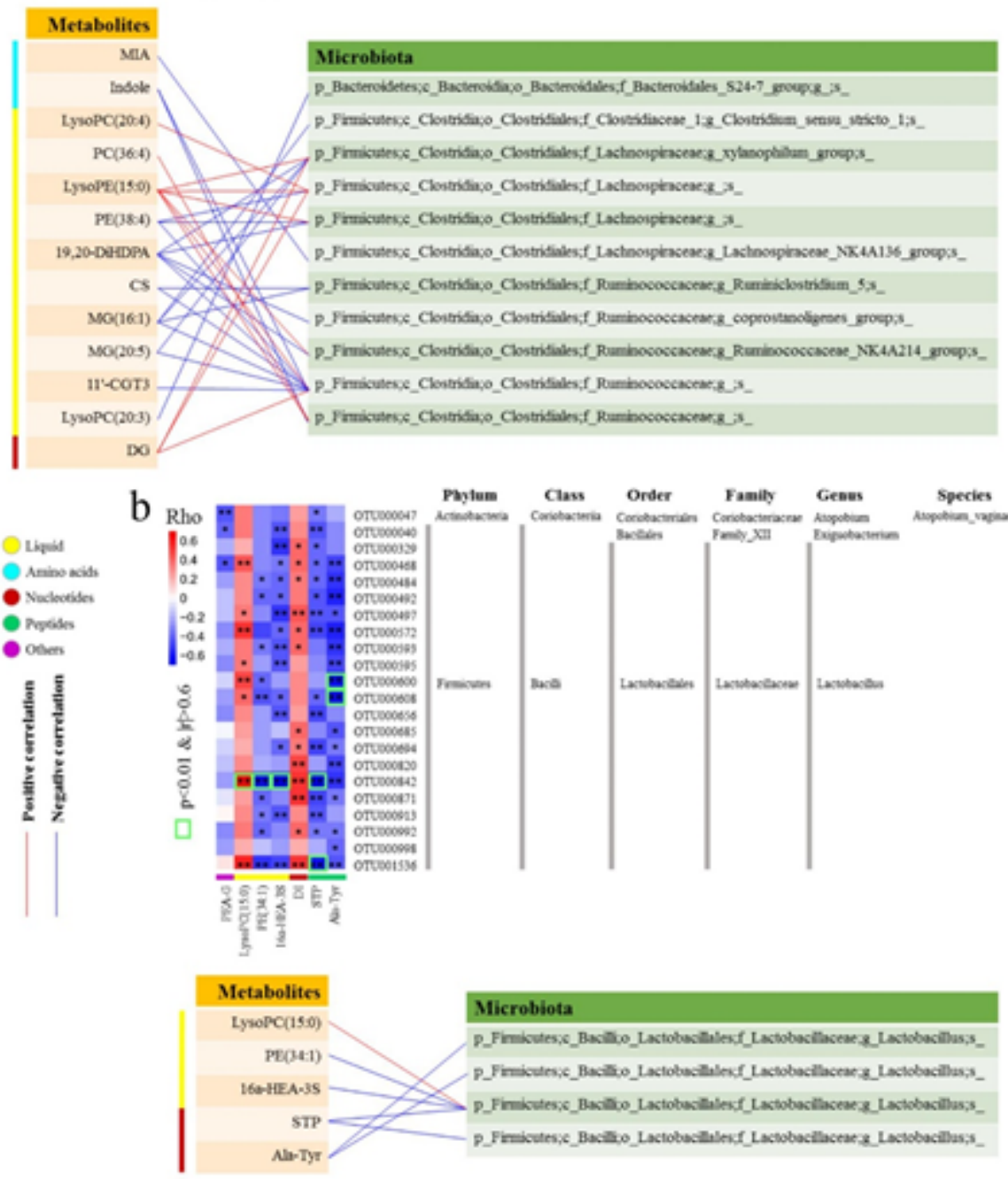

Figure 9

Spearman's rank correlation between the specific gut microbial species and the differential metabolites for the As-treated groups (a) and the As-recovery groups (b). 
This is a list of supplementary files associated with this preprint. Click to download.

- Additionalfile1.pdf

- Additionalfile2.xlsx 